\title{
OPEN Variations of risk factors for ischemic stroke and its subtypes in Chinese patients in Taiwan
}

\author{
Chung-Fen Tsai ${ }^{1,2}$, Cathie L. M. Sudlow ${ }^{2}$, Niall Anderson ${ }^{3}$ \& Jiann-Shing Jeng ${ }^{4 凶}$
}

Chinese have a higher stroke incidence and a different distribution of ischemic stroke (IS) subtypes as compared with Caucasians. Herein we aimed to investigate the prevalence and associations of major risk factors in IS and its subtypes in Chinese patients. From 2006 to 2011, we included 4953 acute IS patients consecutively recruited in National Taiwan University Hospital Stroke Registry (mean age 68 years; male 59\%). For each risk factor, we accessed the proportion in all IS patients, and calculated odds ratios for each main IS subtype versus other subtypes. Multiple logistic regression models were used to adjust for confounders, and to examine the associations of risk factors with IS subtypes. Compared with other ischemic subtypes, large artery atherosclerotic and lacunar strokes were associated with hypertension, diabetes, and hyperlipidaemia, while cardioembolic strokes were associated with ischemic heart disease. Furthermore, the associations with hypertension and diabetes became stronger in lacunar strokes after adjusting for confounders, but not in other ischemic subtypes. Here we report the variable effects of risk factors on different IS subtypes in Chinese patients in Taiwan. Our findings could help shed light on different mechanisms of IS subtypes and provide targets to make more effective strategies for IS prevention.

Although incidence and mortality of stroke tend to decrease in recent 20 years, the global stroke burden continues to increase, especially in developing countries ${ }^{1}$. Compared with western populations, Chinese have a higher incidence and mortality of stroke, along with high disability-adjusted life-years ${ }^{2}$. The stroke burden on Chinese populations is quite heavy, particularly from ischemic stroke (IS) ${ }^{3,4}$.

Our previous systematic review has demonstrated a younger onset, and a different distribution of IS subtypes, especially a higher proportion of lacunar infarct (LI) in Chinese versus Caucasian patients ${ }^{5}$. Yet questions such as what cause the differences remain unanswered. They may relate to differences in genetics, prevalence and associations of risk factors with IS subtypes between populations ${ }^{6-8}$. Further meta-analyses have shown that Chinese IS patients have similar prevalence of hypertension, diabetes, smoking and alcohol, but lower prevalence of atrial fibrillation (AF), ischemic heart disease (IHD) and hypercholesterolemia as compared with Caucasian patients, while associations of risk factors with IS subtypes were similar 9 . However, these findings could be confounded by age, sex, and other risk factors. In addition, they may be driven by risk factor dependent nature of the classification system.

To overcome the confounding effects, and to test the hypothesis that risk factors varied among IS subtypes, we conducted individual patient analyses using data from the National Taiwan University Hospital (NTUH) Stroke Registry. We aimed to evaluate the risk factors distribution in all IS and subtypes, to assess the associations with each main IS subtype versus others using both risk-factor dependent and risk-factor free classification schemes, and to adjust for confounders.

\section{Methods}

Subjects. We included 4953 acute IS Chinese patients from the NTUH Stroke Registry, which had prospectively recruited consecutive acute stroke patients with onset in 10 days. The mean age was 68.1 ( \pm 13.8 ) years; $59 \%$ were men. Informed consent was obtained from all participants or their families ${ }^{10}$. Neurologists assessed the patients, collected medical history and clinical information, and arranged timely brain computed tomography (CT)/magnetic resonance imaging (MRI). The stroke registry and this study protocol had been approved by the NTUH and Cardinal Tien Hospital Institutional Review Boards, and all research was performed in accord-

${ }^{1}$ Department of Neurology, Cardinal Tien Hospital, Fu Jen Catholic University, New Taipei City, Taiwan. ${ }^{2}$ Centre for Clinical Brain Sciences, University of Edinburgh, Edinburgh, UK. ${ }^{3}$ Centre for Population Health Sciences, University of Edinburgh, Edinburgh, UK. ${ }^{4}$ Stroke Center and Department of Neurology, National Taiwan University Hospital, No 7, Chung-Shan South Road, Taipei 100, Taiwan. ${ }^{\circledR}$ email: chungfentsai@gmail.com; jsjeng@ntu.edu.tw 
ance with relevant guidelines/regulations. Herein, we analysed acute IS adult patients from January 2006 to December 2011.

Diagnosis of ischemic stroke and its subtypes. The diagnosis of IS was based on the definition from World Health Organization, and confirmed by brain CT/MRI ${ }^{11}$. IS patients were classified into five etiological subtypes according to the TOAST criteria (risk-factor dependent)-large artery atherosclerosis (LAA: infarct lesions greater than $1.5 \mathrm{~cm}$ on CT/MRI with more than $50 \%$ stenosis of an intracranial or extracranial artery by duplex imaging or CT angiography (CTA)/MR angiography (MRA)/angiography, and excluding cardiogenic embolism), LI (lacunar syndrome with infarct lesion less than $1.5 \mathrm{~cm}$ on brain CT/MRI, and excluding cardiac embolism and large artery stenosis more than 50\% ), cardioembolism (CE: at least one cardiac source of emboli such as atrial fibrillation being identified, and excluding large artery atherosclerosis), other determined, and undetermined etiology. We also classified these IS patients into four anatomical subtypes of the Oxfordshire Community Stroke Project (OCSP classification) - LI, total and partial anterior circulation infarct (TACI and $\mathrm{PACI})$, and posterior circulation infarct (POCI $)^{12,13}$. The OCSP classification system is a risk-factor free scheme based on clinical features of stroke syndrome and brain image findings, being free of etiological assumptions of risk factors to reduce the possibility of classification bias. Patients with previous stroke and its complications, intracerebral/subarachnoid/subdural/epidural haemorrhage, tumor, non-cerebrovascular causes or no brain imaging were excluded.

Major risk factor definitions. Major risk factors were defined as follows: hypertension (history, antihypertensive treatment or blood pressure $\geq 140 / 90 \mathrm{mmHg} 7$ days after stroke), diabetes (history, anti-diabetic treatment, or fasting plasma glucose $\geq 126 \mathrm{mg} / \mathrm{dl}$ ), AF (history or electrocardiographic evidence), IHD (history or electrocardiographic evidence), hyperlipidemia (history, anti-hyperlipidemic medication, hypercholesterolemia or hypertriglyceridemia $\geq 200 \mathrm{mg} / \mathrm{dl}$ ), current or previous smoking, and alcohol intake (habitual drinking more than once per week). We also recorded previous stroke and history of transient ischemic attack (TIA).

Statistical analysis. We used analysis of variance (ANOVA) to compare mean age, and chi-square test to compare proportions of male sex and risk factors among IS subtypes. We divided age into five equal-sized categories as it was not assumed to contribute a linear change, and calculated proportions of risk factors in each IS subtype. For each risk factor, we computed crude odds ratio (OR) with 95\% confidence interval (CI) for LAA, LI, and CE versus other subtypes in the TOAST classification, and used logistic regression to adjust for age and sex and to examine if there was any interaction, obtaining adjusted ORs (OR I). Then we developed a second logistic regression model to adjust for age, sex, and all major risk factors, and used stepwise selection to incorporate the significant 2 -way interactions among variables $(p<0.001)$, obtaining adjusted ORs (OR II). Also, we categorized OCSP subtypes into LI and non-lacunar infarct (non-LI), doing the same analyses for LI versus non-LI.

For TOAST IS subtypes, we did not include AF in the second logistic regression to avoid classification bias as IS patients with AF were most likely assigned to CE subtype. If there was a significant interaction among variables, we conducted further subgroup analyses to exam risk factor associations in different groups. All the statistical hypothesis tests were two-sided, and $p$ values less than 0.05 were regarded as significant. Statistical analyses were performed with $\mathrm{R}^{14}$.

\section{Results}

Characteristics of overall IS patients. We included 4953 acute IS Chinese patients into current analyses, and all had brain CT and electrocardiography (EKG). For cerebrovascular evaluation, around $60 \%$ of these patients had extracranial and intracranial CTA/MRA/angiography, while others had Carotid duplex imaging and transcranial color-coded ultrasonography. Cardiac sonography and 24-h EKG monitoring were performed in patients had cardiac disease, electrocardiography abnormality, suspected embolic stroke on brain image, sudden onset or young age of stroke, or unclear cause of stroke. The mean age was $68.1( \pm 13.8)$ years; $59 \%$ were men. In our study, male patients had younger onset of stroke (mean age: men $66.5 \pm 13.8$ years, women $70.4 \pm 13.6$ years, $p<0.001$ ), more smoking and alcohol intake, but less diabetes, AF and hyperlipidemia than female patients. The clinical characteristics and risk factor distributions were shown in Table 1.

Risk factors for the TOAST subtypes. LI was the most common subtype in the TOAST classification (27.5\%), followed by CE (24.3\%), undetermined (22.1\%), LAA (21.0\%), and other determined etiology $(5.0 \%)$ (Table 2). There were significant age and sex variations among subtypes $(p<0.001)-C E$ patients were the eldest (mean $72.5 \pm 13.2$ years), while LAA had the highest proportion of men (67\%). Different distributions were significant in all vascular risk factors studied among IS subtypes $(p<0.001)$.

Compared with other IS subtypes, LAA strokes were associated with all risk factors studied (Fig. 1). After adjusting for age, sex and other risk factors, hypertension, diabetes, TIA, and hyperlipidemia had more significant associations with LAA versus others. The fully adjusted OR for hypertension was 1.94 (95\% CI 1.59-2.38, $p<0.001$ ), for diabetes was 1.52 (95\% CI 1.32-1.75, $p<0.001$ ), for TIA was 1.72 (95\% CI 1.24-2.36, $p=0.001$ ), and for hyperlipidemia was 1.42 (95\% CI 1.22-1.64, $p<0.001)$. There was no significant interaction among variables.

CE strokes were more associated with age and IHD in comparison with other subtypes (Fig. 2), while having lower prevalence of male sex, diabetes, hyperlipidemia and smoking. There was a strongly significant interaction between IHD and age $(p<0.001)$. While incorporating this interaction, the fully adjusted OR for IHD was 6.74 (95\% CI 4.16-10.87, $p<0.001)$.

For LI versus other IS subtypes, unadjusted analyses showed significant associations with hypertension, diabetes, and hyperlipidemia (Fig. 3), with significant interactions between age and hypertension, and between 


\begin{tabular}{|c|c|c|c|c|c|c|c|}
\hline \multirow[b]{3}{*}{ Mean Age (Y) } & \multicolumn{2}{|l|}{ All } & \multicolumn{2}{|l|}{ Male } & \multicolumn{2}{|c|}{ Female } & \multirow{3}{*}{$\begin{array}{l}p \text {-value } \\
p<0.001\end{array}$} \\
\hline & \multicolumn{2}{|c|}{$68.1( \pm 13.8)$} & \multicolumn{2}{|c|}{$66.5( \pm 13.8)$} & \multicolumn{2}{|c|}{$70.4( \pm 13.6)$} & \\
\hline & $\mathrm{N}$ & (\%) & $\mathbf{N}$ & $(\%)$ & $\mathbf{N}$ & $(\%)$ & \\
\hline & \multicolumn{2}{|l|}{4953} & 2929 & 59.1 & 2024 & 40.9 & $p<0.001$ \\
\hline Hypertension & 3809 & $76.9 \%(75.7-78.7 \%)$ & 2226 & 76.0 & 1583 & 78.2 & $p=0.069$ \\
\hline Diabetes & 1838 & $37.1 \%(35.8-38.4 \%)$ & 1052 & 35.9 & 786 & 38.8 & $p=0.037$ \\
\hline Atrial fibrillation & 1180 & $23.8 \%(22.6-25.0 \%)$ & 616 & 21.0 & 564 & 27.9 & $p<0.001$ \\
\hline Ischemic heart disease & 759 & $15.3 \%(14.3-16.4 \%)$ & 474 & 16.2 & 285 & 14.1 & $p=0.044$ \\
\hline Hyperlipidemia & 1744 & $35.2 \%(33.9-36.6 \%)$ & 990 & 33.8 & 754 & 37.3 & $p=0.012$ \\
\hline Smoking & 1455 & $29.4 \%(28.1-30.7 \%)$ & 1382 & 47.2 & 73 & 3.6 & $p<0.001$ \\
\hline Alcohol & 689 & $13.9 \%(13.0-14.9 \%)$ & 654 & 22.3 & 35 & 1.7 & $p<0.001$ \\
\hline Previous stroke & 1175 & $23.7 \%(22.5-24.9 \%)$ & 707 & 24.1 & 468 & 23.1 & $p=0.409$ \\
\hline Transient ischemic stroke & 193 & $3.9 \%(3.4-4.5 \%)$ & 131 & 4.5 & 62 & 3.1 & $p=0.012$ \\
\hline
\end{tabular}

Table 1. Study characteristics and risk factor distributions in ischemic stroke patients in NTUH Stroke Registry. NTUH National Taiwan University Hospital, Y year, N number.

\begin{tabular}{|c|c|c|c|c|c|c|c|c|c|c|c|}
\hline & \multicolumn{2}{|c|}{$\begin{array}{l}\text { Lacunar } \\
\text { infarct } \\
(\mathrm{N}=1364)\end{array}$} & \multicolumn{2}{|c|}{$\begin{array}{l}\text { LAA } \\
(N=1041)\end{array}$} & \multicolumn{2}{|c|}{$\begin{array}{l}C E \\
(N=1203)\end{array}$} & \multicolumn{2}{|c|}{$\begin{array}{l}\text { Other } \\
\text { determined } \\
(\mathrm{N}=248)\end{array}$} & \multicolumn{2}{|c|}{$\begin{array}{l}\text { Undetermined } \\
(\mathrm{N}=1097)\end{array}$} & \multirow[b]{2}{*}{$p$ value } \\
\hline & $\mathbf{N}$ & $(\%)$ & $\mathbf{N}$ & $(\%)$ & $\mathbf{N}$ & $(\%)$ & $\mathbf{N}$ & $(\%)$ & $\mathbf{N}$ & $(\%)$ & \\
\hline Frequency & \multicolumn{2}{|c|}{$27.5 \%$} & \multicolumn{2}{|c|}{$21.0 \%$} & \multicolumn{2}{|c|}{$24.3 \%$} & \multicolumn{2}{|c|}{$5.0 \%$} & \multicolumn{2}{|c|}{$22.1 \%$} & $p<0.001$ \\
\hline Mean age $(\mathrm{Y})$ & \multicolumn{2}{|c|}{$67.8( \pm 11.8)$} & \multicolumn{2}{|c|}{$70.1( \pm 11.4)$} & \multicolumn{2}{|c|}{$72.5( \pm 13.2)$} & \multicolumn{2}{|c|}{$48.4( \pm 14.4)$} & \multicolumn{2}{|c|}{$66.2( \pm 14.6)$} & $p<0.001$ \\
\hline Sex (male) & 848 & 62.2 & 694 & 66.7 & 624 & 51.9 & 163 & 65.7 & 600 & 54.7 & $p<0.001$ \\
\hline Hypertension & 1124 & 82.4 & 908 & 87.2 & 928 & 77.1 & 115 & 46.4 & 734 & 66.9 & $p<0.001$ \\
\hline Diabetes & 556 & 40.8 & 492 & 47.3 & 379 & 31.5 & 35 & 14.1 & 376 & 34.3 & $p<0.001$ \\
\hline Atrial fibrillation & 41 & 3.0 & 63 & 6.1 & 1037 & 86.2 & 10 & 4.0 & 29 & 2.6 & $p<0.001$ \\
\hline Ischemic heart disease & 96 & 7.0 & 193 & 18.5 & 337 & 28.0 & 11 & 4.4 & 122 & 11.1 & $p<0.001$ \\
\hline Hyperlipidemia & 634 & 46.5 & 437 & 42.0 & 280 & 23.3 & 68 & 27.4 & 325 & 29.6 & $p<0.001$ \\
\hline Smoking & 434 & 31.8 & 350 & 33.6 & 270 & 22.4 & 82 & 33.1 & 319 & 29.1 & $p<0.001$ \\
\hline Alcohol & 197 & 14.4 & 177 & 17.0 & 134 & 11.1 & 34 & 13.7 & 147 & 13.4 & $p=0.002$ \\
\hline Previous stroke & 296 & 21.7 & 301 & 28.9 & 312 & 25.9 & 34 & 13.7 & 232 & 21.1 & $p<0.001$ \\
\hline Transient ischemic attack & 43 & 3.2 & 61 & 5.9 & 53 & 4.4 & 15 & 6.0 & 21 & 1.9 & $p<0.001$ \\
\hline
\end{tabular}

Table 2. Study characteristics and risk factor distributions in ischemic stroke patients in the TOAST classification. $Y$ year, $N$ number, $L A A$ large artery atherosclerosis, $C E$ cardioembolism.

hypertension and diabetes $(p<0.001)$. While incorporating these interactions, the associations with hypertension and diabetes became stronger. The fully adjusted OR for hypertension was 4.84 (95\% CI 3.39-7.03, $p<0.001$ ), and for diabetes was $2.65(95 \%$ CI $1.92-3.65, p=0.025)$. On the contrary, the LI subtype had lower prevalence of IHD (OR II $0.32,95 \%$ CI $0.25-0.40, p<0.001$ ).

Risk factors for the OCSP subtypes. In the OCSP classification, ANOVA showed significant different distributions in age, male sex and all major risk factors studied in different subtypes except TIA (Supplementary Table S1). The mean age was older in TACI (69.6 \pm 15.0 years), while younger in POCI ( $66.7 \pm 13.9$ years). POCI had the highest proportion of men $(63 \%)$, whereas TACI possessed the lowest $(51 \%)$.

In comparison to Non-LI, LI was significantly associated with hypertension (OR II 4.58, 95\% CI 3.16-6.75, $p<0.001$ ), diabetes (OR II 2.40, 95\% CI 1.71-3.37, $p<0.001$ ) and hyperlipidemia (OR II 1.55, 95\% CI 1.34-1.79, $p<0.001$ ), while having lower prevalence of AF (OR II $0.10,95 \%$ CI $0.07-0.13, p<0.001$ ) and IHD (OR II 0.42 , $95 \%$ CI $0.33-0.54, p<0.001$ ) in fully adjusted analysis (Fig. 4 ). There were significant interactions between age and hypertension, and between hypertension and diabetes $(p<0.001)$. The unadjusted and adjusted results for LI versus Non-LI in the OCSP were in line with those in the TOAST classification.

\section{Discussion}

Our results showed that the proportions of hypertension, diabetes and hyperlipidaemia in our IS patients based in Taiwan were significantly higher, while AF, IHD, smoking and alcohol intake were lower than our previous meta-analyses for Caucasian patients ${ }^{9}$. As for the risk factor associations with IS subtypes, LAA and LI were more associated with hypertension, diabetes, and hyperlipidemia (varied in size), while CE strokes were more associated with IHD as compared with other subtypes. In addition, the associations with hypertension and 
Risk factor

Hypertension CR
OR I
OR II
Diabetes CR
OR I
OR II

Ischemic heart CR

OR I

OR II

Hyperlipidemia CR

OR I

OR II

Smoking CR

OR I

OR II

Alcohol CR

OR I

Previous stroke CR

Previous stroke CR

OR I

OR II
TIA CR

TIA CR

OR I

OR II

$$
0.5
$$

OR

$95 \% \mathrm{Cl}$

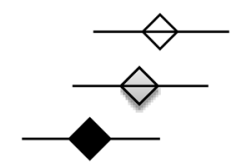

$2.38(1.96,2.92)$

$2.24(1.84,2.74)$

$1.94(1.59,2.38)$

$1.71(1.48,1.97)$

$1.69(1.47,1.94)$

$1.52(1.32,1.75)$

$1.35(1.12,1.62)$

$1.26(1.05,1.51)$

$1.13(0.94,1.36)$

$1.44(1.25,1.66)$

$1.53(1.32,1.76)$

$1.42(1.22,1.64)$

$1.29(1.11,1.49)$

$1.16(0.98,1.37)$

$1.05(0.87,1.27)$

$1.36(1.12,1.67)$

$1.26(1.03,1.54)$

$1.16(0.93,1.44)$

$1.41(1.21,1.65)$

$1.34(1.14,1.56)$

$1.19(1.02,1.40)$

$1.78(1.28,2.45)$

$1.75(1.27,2.39)$

$1.72(1.24,2.36)$

more frequent in other IS subtypes $\leftarrow \rightarrow$ more frequent in the LAA subtype

Figure 1. Risk factor analyses for large artery atherosclerotic strokes versus other ischemic subtypes in TOAST classification. IS = ischemic stroke; LAA = large artery atherosclerosis; $\mathrm{OR}=$ odds ratio; $\mathrm{CR}=$ crude odds ratio; OR I = adjusted odds ratio I; OR II = adjusted odds ratio II; TIA = transient ischemic attack; CI = confidence interval; Horizontal lines represent 95\% CIs. Diamonds represent pooled ORs.

diabetes became stronger in LI after adjusting for confounders, but not in other ischemic subtypes or in Caucasian patients. The analyses in the OCSP classification for LI versus NLI yielded consistent results.

Hypertension and diabetes were independent risk factors for LAA and lacunar strokes in our previous metaanalyses and other report ${ }^{9,15}$. Current study further revealed that hyperlipidemia was also a significant risk factor for both LAA and lacunar strokes in Chinese patients after adjusting for confounding factors. As compared with those in mainland China, our study showed a higher prevalence of these risk factors in IS patients based in Taiwan ${ }^{9,16}$. This finding was also noted in the international REACH registry-a stepwise increase of hypertension, 
Risk factor

Hypertension CR

OR I

OR II

Diabetes CR

ORI

OR II

Ischemic heart CR

OR I

OR II

Hyperlipidemia CR

OR I

OR II

OR

bR II

Alcohol CR

OR I

OR II

Previous stroke CR

ORI

OR II

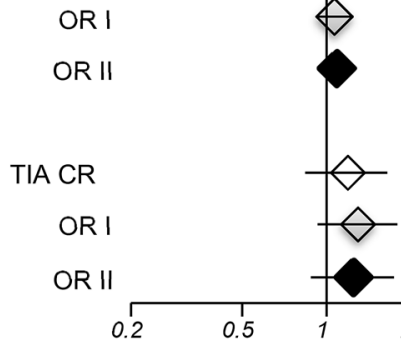

OR

$95 \% \mathrm{Cl}$

$1.02(0.87,1.19)$

$0.85(0.73,1.00)$

$0.91(0.76,1.07)$

$0.72(0.63,0.83)$

$0.70(0.61,0.81)$

$0.67(0.57,0.77)$

3.07 (2.60, 3.62)

$2.78(2.36,3.29)$

$6.74(4.16,10.87)$

$0.47(0.41,0.55)$

$0.51(0.44,0.60)$

$0.51(0.43,0.59)$

$0.63(0.54,0.73)$

$0.81(0.68,0.96)$

$0.78(0.64,0.95)$

$0.72(0.59,0.88)$

$1.00(0.81,1.24)$

$1.14(0.89,1.45)$

$1.17(1.00,1.36)$

$1.07(0.92,1.25)$

$1.09(0.93,1.28)$

$1.19(0.84,1.65)$

$1.30(0.93,1.80)$

$1.25(0.88,1.75)$

more frequent in other IS subtypes $t \rightarrow$ more frequent in the CE subtype

Figure 2. Risk factor analyses for cardioembolic strokes versus other ischemic subtypes in TOAST classification. $\mathrm{IS}=$ ischemic stroke; $\mathrm{CE}=$ cardioembolism; $\mathrm{OR}=$ odds ratio; $\mathrm{CR}=$ crude odds ratio; $\mathrm{OR} \mathrm{I}=$ adjusted odds ratio I; OR II = adjusted odds ratio II; TIA = transient ischemic attack; CI = confidence interval; Horizontal lines represent 95\% CIs. Diamonds represent pooled ORs.

diabetes, and hypercholesterolemia in Chinese patients moving from mainland China to Hong Kong/Singapore/ Taiwan, and to North America/Western Europe ${ }^{17}$, which suggested that westernization of life style and diet habits probably have a heavy impact on these risk factors.

China and Taiwan may represent different stages of epidemiological transition. As infection and nutrition improve, hypertension related disease such as hemorrhagic stroke become more common in the second stage (e.g. mainland China) ${ }^{18}$. When the life expectancy and economics continue improving with more high-fat diet and sedentary life, atherosclerotic diseases such as diabetes and IHD increase in the third stage (e.g. Latin America $)^{18}$. Taiwan is probably also at this stage because of earlier economic development and more influence from western countries. The epidemiological data in Taiwan could be useful to predict the disease evolution in China and other developing countries since they are experiencing rapid economic and lifestyle changes along 
Risk factor

Hypertension CR

OR I

OR II

Diabetes CR

OR I

OR II

Ischemic heart CR

OR I

OR II

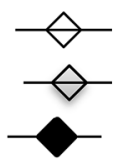

Hyperlipidemia CR

OR I

OR II

Smoking CR

OR I

OR II

Alcohol CR

OR I

OR II
OR

$95 \% \mathrm{Cl}$

$\vartheta$

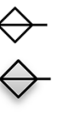

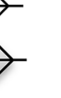

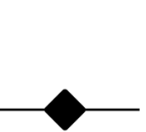

$1.58(1.34,1.86)$

$1.61(1.37,1.89)$

4.84 (3.39, 7.03)

$1.24(1.09,1.41)$

$1.21(1.07,1.38)$

$2.65(1.92,3.65)$

$0.33(0.26,0.42)$

$0.34(0.27,0.42)$

$0.32(0.25,0.40)$

$1.94(1.70,2.21)$

$1.90(1.67,2.16)$

$1.84(1.60,2.10)$

$1.17(1.02,1.35)$

$1.06(0.93,1.27)$

$1.09(0.92,1.30)$

$1.06(0.88,1.27)$

$0.95(0.79,1.15)$

$0.86(0.70,1.07)$

$0.85(0.73,0.99)$

$0.86(0.74,1.00)$

$0.83(0.71,0.97)$

OR II

Previous stroke CR

OR I
OR II

TIA CR

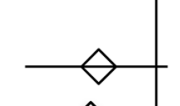

$0.75(0.52,1.06)$

ORI

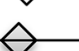

$0.72(0.51,1.01)$

OR II

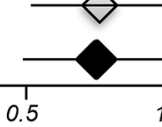

0.2

0.5

more frequent in other IS subtypes $\leftarrow \rightarrow$ more frequent in lacunar infarct

Figure 3. Risk factor analyses for lacunar infarct versus other ischemic subtypes in TOAST classification. $\mathrm{IS}=$ ischemic stroke; $\mathrm{OR}=$ odds ratio; $\mathrm{CR}=$ crude odds ratio; $\mathrm{OR} \mathrm{I}=$ adjusted odds ratio $\mathrm{I}$; OR II = adjusted odds ratio II; TIA = transient ischemic attack; $\mathrm{CI}=$ confidence interval; Horizontal lines represent 95\% CIs. Diamonds represent pooled ORs.

with aging populations. As the prevalence of hypertension, diabetes, and hyperlipidemia have increased in Chinese populations in recent decades, more effective strategies through various education and intervention projects are of vital importance to reduce these risk factors and associated cerebrovascular diseases ahead ${ }^{2,19-21}$.

LI was the most common IS subtype in Chinese stroke patients ${ }^{5}$. In our analyses, there were significant interactions between age and hypertension, and between hypertension and diabetes. The associations with hypertension and diabetes became stronger for LI after incorporating the significant interactions in fully adjusted analyses in in both risk-dependent TOAST and risk-free OCSP classifications, but not in LAA or CE subtypes, or western patients ${ }^{9}$. These findings suggested hypertension and diabetes are strong and important risk factors for LI in Chinese patients. Our findings were different from western research, which showed no difference of hypertension 
Risk factor

Hypertension CR

OR I

OR II

Diabetes CR

OR I

OR II

Atrial fibrillation $\mathrm{CR}$

OR I

OR II

Ischemic heart CR

OR I

OR II

Hyperlipidemia CR

OR I

OR ॥

Smoking CR

OR I

OR II

Alcohol CR

OR I

OR II

Previous stroke CR

OR I
OR II
TIA CR
OR I
OR II

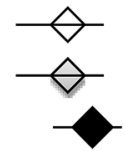

OR

95\% Cl

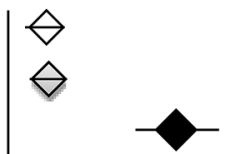

$1.41(1.16,1.67)$

$1.44(1.21,1.70)$

$4.58(3.16,6.75)$

$1.11(0.97,1.27)$

$1.09(0.95,1.25)$

$2.40(1.71,3.37)$

$0.08(0.05,0.11)$

$0.08(0.05,0.11)$

$0.10(0.07,0.13)$

$0.37(0.28,0.46)$

$0.37(0.29,0.47)$

$0.42(0.33,0.54)$

$1.82(1.59,2.09)$

$1.79(1.56,2.05)$

$1.55(1.34,1.79)$

$1.15(0.99,1.33)$

$1.05(0.89,1.23)$

$1.00(0.83,1.21)$

$1.04(0.88,1.26)$

$0.93(0.76,1.14)$

$0.93(0.74,1.16)$

$0.85(0.73,1.00)$

$0.86(0.73,1.00)$

$0.88(0.74,1.04)$

$0.77(0.52,1.11)$

$0.74(0.51,1.06)$

$0.76(0.51,1.11)$

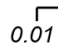

more frequent in non-lacunar infarct $\leftarrow \rightarrow$ more frequent in lacunar infarct

Figure 4. Risk factor analyses for lacunar versus non-lacunar infarct in OCSP classification. OR= odds ratio; $\mathrm{CR}=$ crude odds ratio; OR I = adjusted odds ratio I; OR II = adjusted odds ratio II; TIA = transient ischemic attack; $\mathrm{CI}=$ confidence interval; Horizontal lines represent 95\% CIs. Diamonds represent pooled ORs.

and diabetes between LI and non- $\mathrm{LI}^{22}$, but in line with others studies in eastern Asians ${ }^{23,24}$. The Hisayama study in Japan also reported a greater impact of blood pressure on LI than other IS subtypes ${ }^{23}$. In Taiwan, the prevalence of hypertension and diabetes in IS patients had increased in the past 10 years, while the proportion 
of taking anti-hypertensive or anti-diabetic medication before stroke decreased ${ }^{19}$. Similar findings were also noted in mainland China, where only a low proportion of patients achieved the target goals of control ${ }^{16}$. This may be responsible (at least in part) for a higher proportion of LI in Chinese IS patients and a higher incidence of stroke in Chinese populations. Difference in genetics among ethnic groups, variations of risk factor control, and different methods of case ascertainment may account for these disparities ${ }^{6-9,19,20}$.

As for CE subtype, it had a totally different risk factor distribution. Our results showed CE strokes were more associated with IHD, while having lower prevalence of male sex, diabetes, hyperlipidemia, and smoking. In our analyses for TOAST IS subtypes, we did not include AF in the second logistic regression to avoid classification bias. Even though the classification system does not define subtypes explicitly based on risk factors, there is a tendency for clinicians to bias their assessments according to the strong risk factors present. For example, IS patients with AF are usually assigned to CE subtype, irrespective of other investigation results. In our study, the proportion of CE strokes was higher than previous meta-analyses for the whole Chinese patients or earlier studies, which was probably related to aging population and increasing detection of AF by echocardiography and 24-h holter monitoring ${ }^{5,9,25}$. The incidence of $\mathrm{AF}$ and related strokes are expected to increase ahead because of longer life expectancy and improved survival of IHD, but anticoagulation is still not adequate, especially in Asians ${ }^{25,26}$. Proper holter monitor and adequate anticoagulation may reduce the burden from CE strokes.

Our study documented various influences of risk factors on IS subtypes in Chinese populations, who had a higher incidence of stroke, a different distribution of IS subtypes, especially a higher proportion of lacunar infarct than Caucasians. Furthermore, our results showed that hypertension and diabetes were strong and important risk factors for lacunar stroke in Chinese patient. It had several strengths. First, NTUH Stroke Registry was a well-established registry, prospectively recruiting consecutive acute stroke patients with comprehensive records, without selection of age, sex or socioeconomic status as the Taiwan National Health Insurance provided affordable and compulsive medical care, covering $99 \%$ people in Taiwan. This study benefited from a relatively large number of patients, and detailed information of risk factors. Second, the inclusion of acute stroke patients was based on a standard stroke definition, having timely Neurologist and brain imaging evaluation, and in-depth investigations. Third, we used multiple logistic regressions to adjust for possible confounders, and incorporated significant interactions among variables. There were some limitations. NTUH registry was a large hospital-based stroke registry in Taiwan, which might not be fully representative of all Chinese IS patients. Also, not all acute IS patients received cardiac echography and 24-h EKG monitoring, and only few patients had prolonged EKG monitoring up to $72 \mathrm{~h}$ or longer. Finally, risk factors were collected from medical records before stroke, and from the included patients and their families. Whilst most patients had previous medical records, we could not totally exclude recall bias in a few patients who had no available data before stroke.

Accumulating evidence has suggested that the distributions of IS subtypes and risk factors vary among ethnicities and geographical areas, and there are different associations of risk factors with ischemic subtypes ${ }^{2,5,9,15,22-24}$. While genetics may play a certain role on ischemic subtypes, it is still of vital importance to target the modifiable risk factors through well-designed education and intervention projects. Herein we report various influences of risk factors on main IS subtypes in Chinese IS patients based in Taiwan. As IS accounts for the majority of heavy stroke burden in Chinese populations and around the world, our findings could help shed light on various impacts of risk factors on ischemic subtypes, reveal the potential targets to improve further, and reduce the stroke burden in the years ahead.

\section{Data availability}

The datasets for this study are available from the corresponding author on reasonable request.

Received: 17 November 2020; Accepted: 19 April 2021

Published online: 06 May 2021

\section{References}

1. Feigin, V. L., Norrving, B. \& Mensah, G. A. Global burden of stroke. Circ. Re. 120, 439-448 (2017).

2. Wu, S. et al. Stroke in China: advances and challenges in epidemiology, prevention and management. Lancet Neurol. 18, 394-405 (2019).

3. Wang, W. et al. Prevalence, incidence and mortality of stroke in China: results from a Nationwide Population-Based Survey of 480 687 Adults. Circulation 135, 759-771 (2017).

4. Zhao, D. et al. Epidemiological transition of stroke in China - Twenty-one-year observational study from the Sino-MONONICABeijing Project. Stroke 39, 1668-1674 (2008).

5. Tsai, C. F., Thomas, B. \& Sudlow, C. Epidemiology of stroke and its subtypes in Chinese versus White populations: a systematic review. Neurology 81, 264-272 (2013).

6. NINDS Stroke Genetics Network and International Stroke Genetics consortium. Loci associated with ischaemic stroke and its subtypes (SiGN): a genome-wide association study. Lancet Neurol. 15, 174-184 (2016).

7. Li, J., Luo, M., Xu, X. \& Sheng, W. Association between 1425G/A SNP in PRKCH and ischemic stroke among Chinese and Japanese populations: a meta-analysis including 3686 cases and 4589 controls. Neurosc. Lett. 506, 55-58 (2012).

8. Jackson, C. \& Sudlow, C. Are lacunar strokes really different? A systematic review of differences in risk factor profiles between lacunar and non-lacunar infarcts. Stroke 36, 891-901 (2005).

9. Tsai, C. F., Anderson, N., Thomas, B. \& Sudlow, C. L. M. Risk factors for ischemic stroke and its subtypes in Chinese vs Caucasians: systematic review and meta-analysis. Int. J. Stroke. 10, 485-493 (2015).

10. Jeng, J. S. et al. Subtypes and case-fatality rates of stroke: a hospital-based stroke registry in Taiwan (SCAN-IV). J. Neurol. Sci. 156, 220-226 (1998).

11. Hatano, S. Experience from a multicentre stroke register: a preliminary report. Bull. WHO. 54, 541-553 (1976).

12. Adams HP Jr, et al. Classification of subtype of acute ischemic stroke. Definitions for use in a multicenter clinical trial: TOAST Trial of Org 10172 in Acute Ischemic Stroke. Stroke. 24, 35-41 (1993).

13. Bamford, J.et al. Classification and natural history of clinically identifiable subtypes of cerebral infarction. Lancet 337, 1521-1526 (1991). 
14. R Core Team (2013). R: A language and environment for statistical computing. R Foundation for Statistical Computing, Vienna, Austria. Accessed 23 May, 2019. Available from: http://www.R-project.org/.

15. Arboix, A. et al. Different vascular risk factor profiles in ischemic stroke subtypes: a study from the "Sagrat Cor Hospital of Barcelona Stroke Registry". Acta. Neurol. Scand. 102, 264-270 (2000).

16. Quin $\mathrm{H}$, et al. Management characteristics and prognosis after stroke in China: findings from a large nationwide stroke registry. Stroke and Vascular Neurology. 0, svn-2020-000340 (2020).

17. Chiu JF, et al. REACH Registry Investigators. Cardiovascular risk profiles and outcomes of Chinese living inside and outside China. Eur. J. Cardiovasc. Prev. Rehabil. 17, 668-75 (2010).

18. Yusuf, S., Reddy, S., Ounpuu, S. \& Anand, S. Global burden of cardiovascular diseases: part I: general considerations, the epidemiologic transition, risk factors, and impact of urbanization. Circulation 104, 2746-2753 (2001).

19. Hsieh, C. Y., Wu, D. P. \& Sung, S. F. Trends in vascular risk factors, stroke performance measures, and outcomes in patients with first-ever ischemic stroke in Taiwan between 2000 and 2012. J. Neurol. Sci. 378, 80-84 (2017).

20. Chen, W. W. et al. China cardiovascular diseases report 2015: a summary. J. Geriatr. Cardiol. 14, 1-10 (2017).

21. Feigin, V. L. \& Krishnamurthi, R. Stroke prevention in the developing world. Stroke 42, 3655-3658 (2011).

22. Jackson, C. A. et al. Differing risk factor profiles of ischemic stroke subtypes: evidence for a distinct lacunar arteriopathy?. Stroke 41, 624-629 (2010).

23. Arima, H. et al. Impact of blood pressure levels on different types of stroke: the Hisayama study. J. Hypertens. 27, 2437-2443 (2009).

24. Lai, S. L. et al. Risk factors and subtype analysis of acute ischemic stroke. Eur. Neurol. 50, 230-236 (2008).

25. Pistoia, F. et al. The epidemiology of atrial fibrillation and stroke. Cardiol. Clin. 34, 225-268 (2016).

26. Chao, T. F. et al. Evolving changes of the use of oral anticoagulants and outcomes in patients with newly diagnosed atrial fibrillation in Taiwan. Circulation 138, 1485-1487 (2018).

\section{Acknowledgements}

The authors wish to thank all the staff and participants in National Taiwan University Hospital for their kind help in data collection.

\section{Author contributions}

Study concept and design: C.-F.T., J.-S.J., and C.S. Acquisition of data: J.-S.J. Statistical analysis: C.-F.T., N.A. Interpretation of data: C.-F.T., C.S., and J.-S.J. Manuscript drafting: C.-F.T. Critical revision of the manuscript for important intellectual content: All.

\section{Funding}

This work was partly supported by the Cardinal Tien Hospital Research Grant (CTH 107A-2A09/105A208/109A-2216; C.-F. Tsai), which had no role in study design, data collection and analysis, decision to publish or preparation and writing of the manuscript. Other authors did not receive funding for this work.

\section{Competing interests}

The authors declare no competing interests.

\section{Additional information}

Supplementary Information The online version contains supplementary material available at https://doi.org/ 10.1038/s41598-021-89228-x.

Correspondence and requests for materials should be addressed to C.-F.T. or J.-S.J.

Reprints and permissions information is available at www.nature.com/reprints.

Publisher's note Springer Nature remains neutral with regard to jurisdictional claims in published maps and institutional affiliations.

(c) (i) Open Access This article is licensed under a Creative Commons Attribution 4.0 International License, which permits use, sharing, adaptation, distribution and reproduction in any medium or format, as long as you give appropriate credit to the original author(s) and the source, provide a link to the Creative Commons licence, and indicate if changes were made. The images or other third party material in this article are included in the article's Creative Commons licence, unless indicated otherwise in a credit line to the material. If material is not included in the article's Creative Commons licence and your intended use is not permitted by statutory regulation or exceeds the permitted use, you will need to obtain permission directly from the copyright holder. To view a copy of this licence, visit http://creativecommons.org/licenses/by/4.0/.

(C) The Author(s) 2021 